\title{
Wake-up Stroke and Stroke within the Therapeutic Window for Thrombolysis Have Similar Clinical Severity, Imaging Characteristics, and Outcome
}

\author{
Ricardo Costa, MSc, ${ }^{*}$ João Pinho, MD,† José Nuno Alves, MSc, $†$ \\ José Manuel Amorim, MSc, $\ddagger$ Manuel Ribeiro, MD, $\ddagger$ and Carla Ferreira, MD†
}

\begin{abstract}
Background: Wake-up stroke (WUS) represents $25 \%$ of all ischemic strokes. There is conflicting evidence concerning clinical severity, imaging characteristics, and outcome when WUS is compared with stroke of known time of onset. Our aim was to compare WUS patients with patients with ischemic stroke within the therapeutic window (STW) for thrombolysis. Methods: This is a retrospective hospitalbased study of all consecutive patients hospitalized for acute ischemic stroke during 2013. Patients with STW, WUS, and WUS with computed tomography (CT) at 3 hours or less after awakening (WUS $\leq 3 \mathrm{~h}$ ) were selected for the study. The methods used include a review of clinical records, an independent quantification of early signs of ischemia on admission CT scan, and determination of functional outcome on follow-up. Results: Of 554 patients evaluated, 190 had STW, 113 had WUS (20.4\%), and 25 had WUS $\leq 3 \mathrm{~h}$. Among all WUS patients, 33.6\% did not have any other formal contraindication for thrombolysis besides undetermined time of onset. WUS patients had demographic characteristics, vascular risk factors, and clinical severity similar to STW patients. Mild or absent early signs of ischemia on admission $\mathrm{CT}$ in WUS $\leq 3 \mathrm{~h}$ patients were similar to those in STW patients when adjusted for clinical severity (odds ratio $[\mathrm{OR}]=.50,95 \%$ confidence interval $[\mathrm{CI}]=.17-1.47$ ). Favorable prognosis in WUS $\leq 3 \mathrm{~h}$ was similar to STW when adjusted for age, clinical severity, and thrombolysis ( $\mathrm{OR}=.53,95 \% \mathrm{CI}=.09-3.14)$. Conclusions: This study strengthens the evidence that clinical and early imaging characteristics of WUS patients are similar to those of patients with stroke who are eligible for thrombolysis based on the time window criteria, and patients with WUS do not have a worse short outcome. Key Words: Wake-up stroke—ischemic stroke—computed tomography—stroke outcome.
\end{abstract}

(C) 2016 National Stroke Association. Published by Elsevier Inc. All rights reserved.

From the *School of Health Sciences, University of Minho, Portugal; †Neurology Department, Hospital de Braga, Portugal; and $\ddagger$ Neuroradiology Department, Hospital de Braga, Portugal.

Received March 21, 2015; revision received September 25, 2015; accepted October 31, 2015.

Address correspondence to João Pinho, Hospital de Braga, Sete Fontes, São Victor, 4710-243 Braga, Portugal. E-mail: jdpinho@ gmail.com.

1052-3057/\$ - see front matter

(C) 2016 National Stroke Association. Published by Elsevier Inc. All rights reserved.

http://dx.doi.org/10.1016/j.jstrokecerebrovasdis.2015.10.032

\section{Introduction}

Wake-up stroke (WUS) represents up to $25 \%$ of all ischemic strokes, ${ }^{1}$ and unknown onset of stroke symptoms with time since last seen well for more than 4 hours and 30 minutes is considered a contraindication for thrombolysis. There is an increasing interest in research to define whether WUS patients benefit from reperfusion therapies in the acute phase, and randomized controlled trials are being conducted. ${ }^{2,3}$ Studies comparing characteristics of WUS and stroke with known time of onset are not unanimous, ${ }^{4}$ but can help in selecting inclusion and exclusion criteria for therapeutic trials. The aim of this study was to compare clinical and computed tomography 
C(CT) findings of patients with WUS and patients with stroke of known time of onset and within the therapeutic window for thrombolysis.

\section{Methods}

In this retrospective hospital-based study, records of all consecutive patients hospitalized for acute ischemic stroke during 2013 were reviewed. The patients were categorized in the following groups: clear description of presence of symptoms on awakening was characterized as "WUS"; registered time of onset and CT scan at 4 hours and 30 minutes or less after symptom onset was characterized as "stroke within the therapeutic window" (STW); WUS with time from awakening to CT at 3 hours or less was characterized as "WUS $\leq 3 \mathrm{~h}$. Patients with known onset-to-CT time of more than 4 hours and 30 minutes, unknown onset, and no register of time of onset were excluded. Information on time since last known normal in patients with WUS was not systematically available in the clinical records, except for patients who had symptoms on awakening but had a time since last known normal to CT of less than 4 hours and 30 minutes. These patients were categorized as STW. Information on demographic data and clinical characteristics was collected. Severity of neurological deficits was quantified by reviewing neurological examination of the patient in the emergency department, systematically performed by neurologists in our hospital. Systematic information for scoring National Institutes of Health Stroke Scale (NIHSS) was unavailable, and a mini-NIHSS was applied, in which the item "8. Sensory" was simplified $(0=$ normal, $1=$ mild to total sensory lost), and the following items were omitted: "1b.Level of consciousness questions," "1c.Level of consciousness commands," "2.Best gaze," and "7.Limb ataxia." All admission CT scans were independently reviewed by 2 neuroradiologists, aware of the arterial territory involved and the side of the ischemic stroke, and unaware of clinical deficits. Alberta Stroke Program Early CT Score (ASPECTS), ${ }^{5}$ in the case of carotid territory stroke, and posterior circulation ASPECTS (pcASPECTS), ${ }^{6}$ in the case of vertebrobasilar territory stroke, were evaluated, and disagreements were settled by consensus. Interobserver agreement was determined using Cohen's kappa coefficient. Absent or mild early signs of ischemia on admission CT were considered as an ASPECTS of 7 or more and a pcASPECTS of 7 or more. Etiology was classified according to the Trial of Org 10172 in Acute Stroke Treatment. ${ }^{7}$ Modified Rankin scale (mRS) scores were collected from outpatient clinic records of follow-up visits; an mRS score of 2 or lower was considered a favorable outcome. Distribution of continuous variables was analyzed using the Kolmogorov-Smirnov test. The Pearson chi-square, the Fisher exact test, and the Mann-Whitney test were used to determine group differences in demographic and clinical variables, depending on the comparison and test assumptions. A binary logistic regression model was developed for analyzing ASPECTS/pcASPECTS and follow-up mRS scores in the WUS $\leq 3 \mathrm{~h}$ and STW groups, adjusting for relevant variables. The standard noncorrected significance level of $P$ less than .05 was used. Statistical analysis was performed using SPSS Statistics version 20.0 (IBM Corporation, New York, United States of America)

\section{Results}

Five hundred fifty-four patients were admitted for acute ischemic stroke during the study period. Among all stroke patients, WUS occurred in 113 patients (20.4\%), 25 of which underwent CT scan at 3 hours or less after awakening (WUS $\leq 3 \mathrm{~h}$ ), and 190 patients had STW for thrombolysis. Two hundred fifty-one patients were excluded: 141 had unknown onset or no record of onset time, and 110 had known onset-to-CT time of more than 4 hours and 30 minutes. Demographic and clinical characteristics are shown in Table 1. WUS and STW patients had a relatively high median age (80 and 78 years, respectively) and high prevalence of classical vascular risk factors. Gender, age, vascular risk factors, and CHA2DS2Vasc score were similar in both groups. There was no significant difference in the distribution of mini-NIHSS scores in the WUS and STW groups, however, WUS patients had more frequent posterior circulation infarcts in Oxford Community Stroke Project classification $(P<.0005)$. Accordingly, vertebrobasilar circulation stroke occurred more frequently in WUS patients $(P<.0005)$. In the 230 patients who had adequate etiological investigation, the most frequent cause for stroke was cardioembolism $(n=143,62.2 \%)$, and etiology was similar in the WUS and STW groups. Median time to follow-up was 135 days (interquartile range $=77.5$ 168.0). There was no difference in prevalence of favorable outcome on follow-up (WUS $=37.8 \%$, STW $=43.8 ; P=.359$ ). For comparing early signs of ischemia in admission CT, the WUS $\leq 3$ h subgroup was compared with the STW group. Interobserver agreement for ASPECTS and pcASPECTS was good (kappa $=.86, P<.0005)$. An ASPECTS of 7 or more or a pcASPECTS of 7 or more was less frequent in the WUS $\leq 3$ h group $(P=.014)$; however, this subgroup had a significantly greater median mini-NIHSS score (Table 1). In the multivariate analysis adjusted for miniNIHSS, WUS $\leq 3$ h was less likely than STW to present ASPECTS $\geq 7$ or pcASPECTS $\geq 7$, but this difference was not significant (odds ratio $=.50,95 \%$ confidence interval $=.17$ $1.47, P=.208$ ) (Table 2). Favorable outcome was analyzed in the WUS $\leq 3 \mathrm{~h}$ group using STW as the reference group, and after adjusting for mini-NIHSS, age and intravenous thrombolysis, no differences were found (odds ratio $=.53,95 \%$ confidence interval $=.09-3.14, P=.486$ ) (Table 2). Among all WUS patients, 38 (33.6\%) had no 
Table 1. Characteristics of the study population

\begin{tabular}{|c|c|c|c|c|c|}
\hline Characteristics & $\begin{array}{c}\text { STW } \\
(\mathrm{n}=190)\end{array}$ & $\begin{array}{c}\text { WUS } \\
(\mathrm{n}=113)\end{array}$ & $\begin{array}{c}P \text { value } \\
(\text { STW versus WUS })\end{array}$ & $\begin{array}{c}\text { WUS } \leq 3 \mathrm{~h} \\
(\mathrm{n}=25)\end{array}$ & $\begin{array}{c}P \text { value } \\
(\text { STW versus } \mathrm{WUS} \leq 3 \mathrm{~h})\end{array}$ \\
\hline Age (years) & $78(69-83)$ & $80(65-85)$ & .150 & $81(68-84)$ & .109 \\
\hline Female sex, $\mathrm{n}(\%)$ & $116(61.1)$ & $71(62.8)$ & .758 & $18(72.0)$ & .288 \\
\hline Arterial hypertension, n (\%) & $149(78.4)$ & $86(76.1)$ & 640 & $17(68.0)$ & .243 \\
\hline Diabetes mellitus, n (\%) & $48(25.3)$ & $30(26.5)$ & .805 & $10(40.0)$ & .119 \\
\hline Dyslipidemia, n (\%) & $92(48.4)$ & $57(50.4)$ & .734 & $12(48.0)$ & .968 \\
\hline $\begin{array}{l}\text { Coronary heart disease/peripheral } \\
\text { atherosclerotic disease, } \mathrm{n}(\%)\end{array}$ & $18(9.5)$ & $8(7.1)$ & .472 & $1(4.0)$ & .321 \\
\hline CHA2DS2Vasc score & $6(4-7)$ & $6(4-6)$ & .212 & $6(4-7)$ & .086 \\
\hline \multicolumn{6}{|l|}{ OCSP } \\
\hline Total anterior circulation infarct & 93 (48.9) & $39(34.5)$ & .014 & $13(52.0)$ & .774 \\
\hline Partial anterior circulation infarct & $49(25.8)$ & $29(25.7)$ & .981 & $6(24.0)$ & .533 \\
\hline Lacunar infarct & $32(16.8)$ & $16(14.2)$ & .536 & $1(4.0)$ & .073 \\
\hline Posterior circulation infarct & $15(7.9)$ & $29(25.7)$ & $<.0005$ & $5(20.0)$ & .064 \\
\hline Mini-NIHSS score & $9(4-16)$ & $7(3-13)$ & .442 & $14(11-18)$ & .005 \\
\hline Carotid artery territory stroke, $\mathrm{n}(\%)$ & $160(84.2)$ & $77(68.1)$ & .001 & $20(80.0)$ & .592 \\
\hline $\begin{array}{l}\text { Vertebrobasilar artery territory } \\
\text { stroke, } \mathrm{n}(\%)\end{array}$ & $21(11.1)$ & $35(31.0)$ & $<.0005$ & $5(20.0)$ & .165 \\
\hline ASPECTS or pcASPECTS $(\geq 7)$ & $159(84.1)$ & $87(79.8)$ & .345 & $16(64.0)$ & .014 \\
\hline Intravenous thrombolysis & $90(47.4)$ & $4(3.5)$ & $<.0005$ & $1(4.0)$ & $<.0005$ \\
\hline \multicolumn{6}{|l|}{ Etiology } \\
\hline Cardioembolism, n (\%) & $92(61.7)$ & $51(63.0)$ & .856 & $13(76.5)$ & .233 \\
\hline Large-vessel disease, $\mathrm{n}(\%)$ & $7(4.7)$ & $6(7.4)$ & .285 & $2(11.8)$ & .223 \\
\hline Small-vessel disease, $\mathrm{n}(\%)$ & $17(1.4)$ & $11(13.6)$ & .631 & $0(0)$ & .144 \\
\hline Cryptogenic, n (\%) & $24(16.1)$ & $12(14.8)$ & .797 & $2(11.8)$ & .481 \\
\hline Follow-up mRS score $(\leq 2), \mathrm{n}(\%)$ & $64(43.8)$ & $34(37.8)$ & .359 & $2(9.1)$ & .001 \\
\hline
\end{tabular}

Abbreviations: ASPECTS, Alberta Stroke Program Early CT Score; CT, computed tomography; mRS, modified Rankin scale; NIHSS, National Institutes of Health Stroke Scale; OCSP, Oxford Community Stroke Project; pcASPECTS, posterior circulation ASPECTS; STW, stroke within the therapeutic window; WUS, wake-up stroke; WUS $\leq 3 \mathrm{~h}$, wake-up stroke with CT scan at 3 hours or less after awakening.

Continuous variables are presented as median (interquartile range).

Table 2. Multivariate analyses using binary logistic regression models

\begin{tabular}{lcc}
\hline \multicolumn{1}{c}{ Variables included in the models } & Odds ratio $(95 \%$ confidence interval) & $P$ value \\
\hline ASPECTS $(\geq 7)$ or pcASPECTS $(\geq 7)$ & $.50(.17-1.47)$ & .208 \\
WUS $\leq 3 \mathrm{~h}$ (STW as reference group) & $.83(.77-.89)$ & .0005 \\
Mini-NIHSS score (for each increment of 1 point) & $.53(.09-3.14)$ \\
mRS score ( $\leq 2)$ & $.96(.93-.99)$ & .004 \\
WUS $\leq 3 \mathrm{~h}$ (STW as reference group) & $.80(.73-.87)$ & .0005 \\
Age (for each increment of 1 year) & $3.59(1.40-9.22)$ & .008 \\
Mini-NIHSS score (for each increment of 1 point) & \\
Intravenous thrombolysis & \\
\hline
\end{tabular}

Abbreviations: ASPECTS, Alberta Stroke Program Early CT Score; mRS, modified Rankin scale; NIHSS, National Institutes of Health Stroke Scale; pcASPECTS, posterior circulation ASPECTS; STW, stroke within the therapeutic window; WUS $\leq 3 \mathrm{~h}$, wake-up stroke with CT scan at 3 hours or less after awakening.

formal contraindication for thrombolysis other than undetermined time of onset.

\section{Discussion}

This study confirms the significant prevalence of WUS (one fifth of all ischemic strokes), which, given the potential eligibility for reperfusion therapies in one third of these patients, could significantly increase the number of patients who benefit from thrombolysis. We found that clinical severity in WUS patients was not different from that in patients with STW. Additionally, signs of early ischemia in CT performed in the hyperacute phase were also similar. Given that we routinely do not subject WUS patients to thrombolysis in our hospital, there was a wide range of time from awakening to $\mathrm{CT}$, and for comparing 
ASPECTS and pcASPECTS, we used a time limit of 3 hours, which was defined as the time limit for thrombolysis in several studies. ${ }^{4}$ Lack of information on the time since last known normal for patients with WUS may limit the interpretation of data and may influence the results of imaging studies. Similar to several ongoing therapeutic trials for WUS, we used the time since symptom recognition to select a subgroup of patients with WUS comparable with patients with STW. Studies suggest that WUS occurs predominantly in the early hours of the morning ${ }^{1,8}$; therefore, the more suitable time point for defining WUS onset and for defining a therapeutic window may be that of symptom recognition rather than time since last known normal. Our findings are in accordance to the evidence that clinical and early CT findings in WUS patients are not different from patients with stroke of known onset, ${ }^{9-13}$ and suggest that WUS may occur predominantly in a time period shortly before awakening. Selection of WUS patients for thrombolysis based on "low-tech" criteria, such as time from awakening-to-needle and quantification of early signs of ischemia in $\mathrm{CT}_{1}^{14}$ although having the limitation of not providing information regarding penumbra or definite core ischemic lesion may be sufficient to guarantee safety of thrombolysis and more advantageous regarding time to treatment, availability, and costeffectiveness. Independent predictors of favorable outcome on short-term follow-up were age, severity of neurological deficits, and thrombolysis, while recognition of symptoms on awakening was not associated with a worse mRS score on univariate (all WUS patients) or multivariate (WUS $\leq 3 \mathrm{~h}$ patients) analysis. The finding of a higher frequency of vertebrobasilar circulation involvement in WUS patients is intriguing and has previously been unreported. ${ }^{9,10}$ This may be related to the fact that patients with minor deficits attributable to small carotid artery territory lesions may have been discharged home from the emergency department and therefore were not included in our initial population of 554 patients. Our study population had a high median age, explained by the fact that we included all consecutive patients with ischemic stroke admitted in our hospital, therefore reflecting daily clinical practice in countries with increasingly older population. The main limitations of the present study are related to its retrospective nature, which precluded collection of systematic information on all NIHSS items for all patients and prompted us to use a simplified version of this scale. Similar modified NIHSS scales have been published, and the validity for their retrospective use has been shown, ${ }^{15,16}$ but their use limits the comparison with other studies that use the standard version of NIHSS. Additional limitations include exclusion of patients with minor strokes who were not hospitalized, nonstandardized followup, and small size population of WUS with early CT for adequate imaging comparison with the control group.

In conclusion, this study shows that not only severity of neurological deficits of WUS patients is similar to stroke patients within the therapeutic window for intravenous thrombolysis, but also frequency of absent or mild early signs of cerebral ischemia on admission CT is comparable in these 2 groups. Therefore, revascularization treatments in the hyperacute phase, either intravenous or intra-arterial, are likely to be safe in WUS patients, and ongoing trials to study the benefit of these treatments in this important group of patients may clarify which are the most adequate clinical and imaging selection criteria.

\section{References}

1. Lago A, Geffner D, Temble J, et al. Circadian variation in acute ischemic stroke: a hospital-based study. Stroke 1998;29:1873-1875.

2. Buck D, Shaw LC, Price CI, et al. Reperfusion therapies for wake-up stroke: systematic review. Stroke 2014; 45:1869-1875.

3. Rimmele DL, Thomalla G. Wake-up stroke: clinical characteristics, imaging findings, and treatment option - an update. Front Neurol 2014;5:1-7. doi:10.3389/fneur .2014 .00035 .

4. Kang D-W, Kwon JY, Kwon SU, et al. Wake-up or unclear onset strokes: are they waking up to the world of thrombolysis therapy? Int J Stroke 2012;7:311-320.

5. Barber PA, Demchuk AM, Zhang J, et al. Validity and reliability of a quantitative computed tomography score in predicting outcome of hyperacute stroke before thrombolytic therapy. Lancet 2000;355:1670-1674.

6. Puetz V, Sylaja PN, Coutts SB, et al. Extent of hypoattenuation on $\mathrm{CT}$ angiography source images predicts functional outcome in patients with basilar artery occlusion. Stroke 2008;39:2485-2490.

7. Adams HP, Bendixen BH, Kappelle LJ, et al. Classification of subtype of acute ischemic stroke. Definitions for use in a multicenter clinical trial. TOAST. Trial of Org 10172 in Acute Stroke Treatment. Stroke 1993;24:35-41.

8. Kelly-Hayes M, Wolf PA, Kase CS, et al. Temporal patterns of stroke onset. The Framingham Study. Stroke 1995;26:1343-1347.

9. Reid JM, Dingwei D, Cheripelli B, et al. Differences in wake-up and unknown onset stroke examined in a stroke registry. Int J Stroke 2014. doi:10.1111/ijs.12388.

10. Silva GS, Lima FO, Camargo EC, et al. Wake-up stroke: clinical and neuroimaging characteristics. Cerebrovasc Dis 2010;29:336-342.

11. Mackey J, Kleindorfer D, Sucharew H, et al. Populationbased study of wake-up strokes. Neurology 2011;76:16621667.

12. Todo K, Moriwaki H, Saito K, et al. Early CT findings in unknown-onset and wake-up strokes. Cerebrovasc Dis 2006;21:367-371.

13. Fink JN, Kumar S, Horkan C, et al. The stroke patient who woke up: clinical and radiological features, including diffusion and perfusion MRI. Stroke 2002;33:988-993.

14. Balucani C, Grotta JC. Selecting stroke patients for intra-arterial therapy. Neurology 2012;78:755-761.

15. Kasner SE, Cucchiara BL, McGarvey ML, et al. Modified National Institutes of Health Stroke Scale can be estimated from clinical records. Stroke 2003;34:568-570.

16. Gonzalez MA, Hanna N, Rodrigo ME, et al. Reliability of prehospital real-time cellular video phone in assessing the simplified National Institutes of Health Stroke Scale in patients with acute stroke: a novel telemedicine technology. Stroke 2011;42:1522-1527. 